\title{
Trasplante simultáneo de páncreas y riñón en diabetes mellitus tipo 1: Experiencia de un centro en Chile
}

\author{
ANDREA ALBA ${ }^{1}$, JORGE MORALES ${ }^{1}$, MARIO FERRARIO ${ }^{1}$, \\ CARLOS ZEHNDER ${ }^{1}$, JORGE AGUILÓ ${ }^{1}$, CARLOS ZAVALA ${ }^{1}$, \\ CRISTINA HERZOG ${ }^{1,2}$, LORENA CALABRAN ${ }^{1,2}$, LUIS CONTRERAS $^{1}$, \\ RICARDO ESPINOZA ${ }^{1}$, ERWIN BUCKEL ${ }^{1}$, JUAN ALBERTO FIERRO ${ }^{1}$
}

\section{Simultaneous kidney and pancreas transplantation (SKPT) in patients with type 1 diabetes and chronic renal failure. Experience in 12 patients in Chile}

Background: Simultaneous kidney and pancreas transplantation (SKPT) is the best alternative for end stage renal disease among patients with insulin dependent diabetes mellitus. Aim: To report our experience with SKPT. Material and Methods: Retrospective analysis of 12 recipients of SKPT transplanted in one center starting in 1994, with a mean follow-up period of 6.8 years (2-15). Results: Eleven of 12 recipients were in chronic hemodialysis before SKPT. Mean A, B, DR and HLA mismatch was 4.3. Mean preformed anti HLA antibodies was $3.3 \%$. Mean cold ischemia times for pancreas and kidney were 6 and 10 hours, respectively. In the first eight cases, the pancreas was drained to the bladder, and in the last four, an enteric drainage was performed. Eleven recipients were induced with antibodies, and maintenance immunosuppression consisted of Cyclosporine or Tacrolimus plus an antiproliferative agent. Ten year patient survival was $70 \%$. Pancreas and kidney survival, defined by insulin and dialysis independence, were 72 and $73 \%$ respectively. Fifty percent of recipients experienced acute graft rejection (cellular or humoral), with good response to treatment except in one case. Conclusions: This experience shows that SKPT is associated with an excellent patient survival associated to insulin and dialysis independence in $70 \%$ of patients at 10 years.

(Rev Med Chile 2011; 139: 11-18).

Key words: Kidney transplantation; Organ transplantation; Pancreas transplantation.

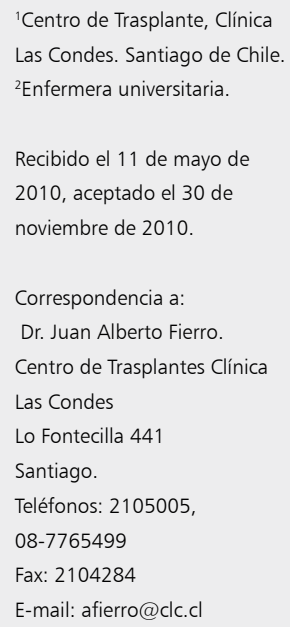

L a diabetes mellitus tipo 1 (DM1) tiene una incidencia en Chile de 4.5/100.000 y una prevalencia de 24/100.000. Las complicaciones sistémicas de la DM1 conducen a una disminución significativa tanto de la calidad como de la esperanza de vida, las que se agravan con la instalación de nefropatía diabética e insuficiencia renal. En DM 1 se observa la aparición de nefropatía diabética en 25 a 30\% de los pacientes. La nefropatía crónica ocurre entre 14 y $30 \%$ de los pacientes con DM1 veinte años después de iniciada la enfermedad ${ }^{1,2}$. En Chile, el $35,4 \%$ de la población que recibe tratamiento de hemodiálisis tiene como enfermedad de base DM 1 ó 2, constituyendo la DM 1 el 3,6\% de la población total en diálisis, lo que corresponde a 416 enfermos en el año 2010 (observaciones no publicadas). La hemodiálisis en pacientes diabéticos se asocia a una mortalidad de más de $50 \%$ en 4 años ${ }^{3}$. 
En 1966 Kelly y cols efectuaron el primer trasplante de páncreas en humanos y desde entonces el procedimiento se ha ido extendiendo hasta llegar a ser terapia de rutina en paises desarrollados en pacientes con nefropatía diabética terminal originada en DM1. El último reporte del Organ Procurement and Transplant Network, (OPTN, USA, http:// www.unos.org/data/) muestra que en USA se han practicado desde 1988 más de 16.000 trasplantes simultaneos de páncreas y riñón (TSPR) y en el año 2008 éstos fueron 837 o sea una tasa de 2,79 TSPR por millón de habitantes ${ }^{4}$. En contraste, la lista de espera de pacientes para TSPR en Chile es mínima, no hay reconocimiento de los sistemas de previsión para el financiamiento del procedimiento y la tasa de TSPR es sólo de 0,06 por millón de habitantes (S. Elgueta. Registro de Trasplante. XXVI Congreso Conjunto de Nefrología, Hipertensión y Trasplante, noviembre de 2009). El objetivo del presente estudio fue conocer los resultados del TSPR realizado en Clínica Las Condes después de un período de observación promedio de 6,8 años. $\mathrm{El}$ análisis retrospectivo se ha centrado en variables objetivas: sobrevida de pacientes e injertos de páncreas y riñón, causas de mortalidad y pérdida de injertos, estado funcional de páncreas y riñón, complicaciones infecciosas importantes, morbilidad vascular e incidencia de cáncer.

\section{Pacientes y Métodos}

Diseño: Estudio unicéntrico retrospectivo, descriptivo, de pacientes sometidos a TSPR.

Receptores: Doce pacientes recibieron TSPR órganos de personas fallecidas, entre los años 1994 y 2007, con un seguimiento promedio de 6,8 años (rango 2 a 15 años). El período final de observación fue el 31 de diciembre de 2008. Se incluyeron pacientes con DM1, nefropatía diabética con insuficiencia renal etapa 4 y 5 , sin enfermedad neoplásica no curada, sin enfermedad infecciosa activa o enfermedad coronaria o carotídea sintomática no corregida. Se exigió compatibilidad de grupo sanguíneo y "crossmatch" negativo para linfocitos T y B. No fue criterio de exclusión para el TSPR la compatibilidad HLA donante receptor.

La edad promedio del receptor al momento del trasplante fue de $38 \pm 9$ años, la distribución por género fue igual para ambos sexos, el tiempo promedio de evolución de la DM1 fue de $22 \pm 8,6$ años, el tiempo promedio de espera en diálisis fue de $10 \pm 6,8$ meses (Tabla 1 ).

Donantes: Criterios de selección de donante de páncreas: edad $<45$ años, ausencia de DM 1 ó 2 , índice masa corporal $<30$, peso $>30 \mathrm{~kg}$, sin cirugía pancreática ni esplenectomía, ausencia de: historia

Tabla 1. Demografia de receptores de trasplante simultáneo de páncreas y riñón

\begin{tabular}{|ccccccccc|}
\hline & $\begin{array}{c}\text { Edad } \\
\text { (años) }\end{array}$ & Género & $\begin{array}{c}\text { Tiempo en } \\
\text { hemodiálisis } \\
\text { (meses) }\end{array}$ & $\begin{array}{c}\text { Requerimiento } \\
\text { insulina } \\
\text { (UI /día) }\end{array}$ & HbA1c & IMC* & $\begin{array}{c}\text { Péptido C } \\
\text { (mg/dl) }\end{array}$ & $\begin{array}{c}\text { PRA** } \\
\text { pre tx*\% }\end{array}$ \\
\hline 1 & 33 & F & 9 & 23 & 9,6 & 23,4 & 0 & 0 \\
\hline 2 & 33 & M & 12 & 10 & & 20,7 & & 8 \\
\hline 3 & 33 & F & 6 & 20 & 9,4 & 21,7 & $<0,2$ & 0 \\
\hline 4 & 26 & M & 4 & 20 & 10,9 & 21,5 & $<0,2$ & 5 \\
\hline 5 & 47 & F & 24 & 14 & 11,0 & 23,5 & 0,9 & 0 \\
\hline 6 & 40 & M & 6 & 30 & 11,1 & 21,1 & $<0,5$ & 0 \\
\hline 7 & 47 & F & 7 & 22 & 8,2 & 15,4 & 0,3 & 0 \\
\hline 8 & 39 & M & 11 & 30 & 12,6 & 21,5 & $<0,5$ & 0 \\
\hline 9 & 32 & F & 16 & 20 & 12,0 & 22,3 & 0,5 & 0 \\
10 & 51 & M & 0 & 14 & 7,2 & 21,9 & $<0,5$ & 0 \\
\hline 11 & 47 & M & 5 & 55 & 18,9 & 29,4 & 0,04 & 27 \\
\hline 12 & 24 & F & 18 & 12 & 9,6 & 20,9 & 0,5 & 0 \\
\hline
\end{tabular}

*Indice de masa corporal. **anticuerpos HLA previos al trasplante; tx: trasplante. 
de pancreatitis aguda o crónica, antecedente de alcoholismo crónico.

\section{Cirugía del trasplante}

El injerto renal fue abocado a vasos ilíacos izquierdos (arteria y vena) con anastomosis término lateral en todos los casos. El uréter del injerto fue implantado a la vejiga con técnica antireflujo de Lich-Gregoire. El páncreas fue abocado a vejiga en 8 casos y a ileon en 4 pacientes. Los tiempos promedio de isquemia fría (TIF) del páncreas y del riñón fueron de 5,6 y 7 horas respectivamente.

\section{Evaluación inmunológica}

El promedio de PRA (anticuerpos HLA previos al trasplante) fue de 3,3\% (PRA máximo 27\%). El promedio de "mismatch" HLA (MM) fue 4,3 (rango 3 a 6).

\section{Inmunosupresión}

Los pacientes recibieron inducción con anticuerpos: Timoglobulina ${ }^{\circledR}$ en 5 casos, basiliximab (Simulect ${ }^{\circledR}$ ) en 3 pacientes y alemtuzumab (Campath-1 $\mathrm{H}^{\circledR}$ ) en 3 casos. Un receptor no recibió inducción. Los receptores fueron tratados con metilprednisolona y luego prednisona en dosis decrecientes; ciclosporina o tacrolimus, asociados a azatioprina, micofenolato mofetil o sirolimus
(Tabla 2). Se efectuó tratamiento profiláctico con ganciclovir o valganciclovir en 6 casos y con aciclovir en 2 receptores; en 4 receptores se controló la viremia CMV con antigenemia periódica. Todos los pacientes recibieron profilaxis para pneumocistis jeroveci con cotrimoxazol por un período de 6 meses y antifúngica con nistatina oral por tres meses.

\section{Evaluación funcional}

La filtración glomerular fue calculada con la fórmula de Cockroft Gault. El diagnóstico de rechazo agudo de trasplante renal fue sospechado en caso de incremento sostenido de la creatinina plasmática igual o mayor a $0,3 \mathrm{mg} / \mathrm{dl}$ en ausencia de factores pre o post renales de disfunción, confirmando el diagnóstico por biopsia renal. El diagnóstico de rechazo de páncreas fue sospechado en caso de disminución de la amilasuria asociado o no a aumento de enzimas pancreáticas en sangre: lipasa y amilasa. El diagnóstico de pancreatitis fue sospechado por síntomas sugerentes asociados a aumento de lipasa y amilasa en sangre. La determinación de péptido $\mathrm{C}$ se hizo con una periodicidad anual. Los niveles de los anticalcineurínicos (IMX Abbot Park, Illinois) fueron determinados periódicamente.

Tabla 2 Características de los donantes y del trasplante simultáneo de páncreas y riñón

\begin{tabular}{|ccccccccc|}
\hline Donante & $\begin{array}{c}\text { HLA Mis } \\
\text { match }\end{array}$ & $\begin{array}{c}\text { Tiempo } \\
\text { isquemia fría } \\
\text { páncreas }\end{array}$ & $\begin{array}{c}\text { Tiempo } \\
\text { isquemia fría } \\
\text { riñón }\end{array}$ & $\begin{array}{c}\text { Anast } \\
\text { páncreas }\end{array}$ & $\begin{array}{c}\text { Diálisis } \\
\text { Post tx }\end{array}$ & $\begin{array}{c}\text { Inmuno } \\
\text { supresión* }\end{array}$ \\
\hline 1 & 50 & F & 3 & 8 & 10 & vejiga & No & Est/aza/CsA \\
2 & 36 & M & 5 & 6 & 8 & vejiga & No & Est/aza/CsA \\
\hline 3 & 12 & M & 4 & 16 & 19 & vejiga & No & Est/aza/CsA \\
4 & 34 & F & 5 & 10 & 12 & vejiga & No & Est/MMF/CsA \\
\hline 5 & 14 & M & 4 & 4 & 5 & vejiga & No & Est/MMF/FK \\
6 & 18 & F & 4 & 3 & 4 & vejiga & No & Est/MMF/FK \\
\hline 7 & 25 & M & 5 & 5 & 3 & vejiga & No & Est/Rapa/FK \\
\hline 8 & 10 & M & 4 & 4 & 3 & vejiga & No & Est/MMF/FK \\
\hline 9 & 25 & F & 3 & 5 & 4 & íleon & No & Est/MMF/FK \\
10 & 33 & M & 4 & 12 & 10 & íleon & No & Est/MMF/FK \\
\hline 11 & 18 & M & 6 & 6 & 8 & íleon & No & Est/MMF/FK \\
\hline 12 & 42 & F & 5 & 4 & 3 & íleon & No & Est/MMF/FK \\
\hline
\end{tabular}

\footnotetext{
* Est, esteroides; aza, azatioprina; CsA, ciclosporina; MMF, micofenolato mofetil; FK, tacrolimus; tx: trasplante.
} 


\section{Estadística}

Se consideró sobrevida de páncreas el período post trasplante en que el paciente estuvo independiente de insulina y sobrevida renal el período después del mes post trasplante que el paciente no requirió tratamiento de diálisis. El fallecimiento con órgano funcionante fue considerado pérdida de ambos injertos. La sobrevida se calculó por el método de Kaplan-Meier usando el programa estadístico SPSS (versión 11.5). Los resultados se expresan en número y porcentaje, promedio y desviación estándar.

\section{Resultados}

\section{Sobrevida de pacientes}

La sobrevida de los pacientes fue de $70 \%$ a los 5 y 10 años (Figura 1). Tres pacientes fallecieron a los 3 años de trasplante por enfermedad coronaria $\mathrm{y}$ vascular periférica severas, septicemia urinaria y pielonefritis fúngica.

\section{Función y sobrevida del páncreas (Tabla 3,} Figura 2)

La independencia de insulina se observó desde la apertura de la arteria esplénica del injerto pancreático en todos los pacientes. Dos receptores (17\%) presentaron pérdida funcional del páncreas por rechazo crónico dos años post trasplante, falleciendo un año después. La sobrevida del páncreas (censado el único caso de fallecimiento con injerto funcionante) fue de $72 \%$ a los 5 y 10 años. Las concentraciones plasmáticas de péptido C fueron en promedio $4,3 \pm 2,2,3,4 \pm 2,8$ y $9,3 \pm$ $11,7 \mathrm{mg} / \mathrm{dl}$ al año, 5 y 10 años.

\section{Función y sobrevida renal (Figura 3)}

Tres pacientes retornaron a diálisis, 2 también perdieron función pancreática y fallecieron posteriormente y uno ingresó a diálisis 12 años post TSPR. La sobrevida del trasplante renal fue de $73 \%$ a los 5 y 10 años. La filtración glomerular fue de $63,8 \pm 23,53,9 \pm 16$ y $35,5 \pm 3 \mathrm{ml} / \mathrm{min}$ en los mismo períodos.

\section{Complicaciones quirúrgicas}

Tres pacientes fueron reoperados por colecciones peri-pancreáticas en el primer mes; un paciente presentó filtración irreparable de la anastomosis vascular arterial del riñón trasplantado que obligó a la nefrectomía del injerto e implante de un segundo riñón del mismo donante. En otro receptor, la derivación pancreática a la vejiga fue transformada en anastomosis pancreo-ileal cinco años post trasplante debido a hematuria recurrente, uretritis y cistitis.

\section{Rechazo agudo}

Hubo 8 episodios de rechazo agudo en 6 pacientes, tratados sólo con metilprednisolona (3

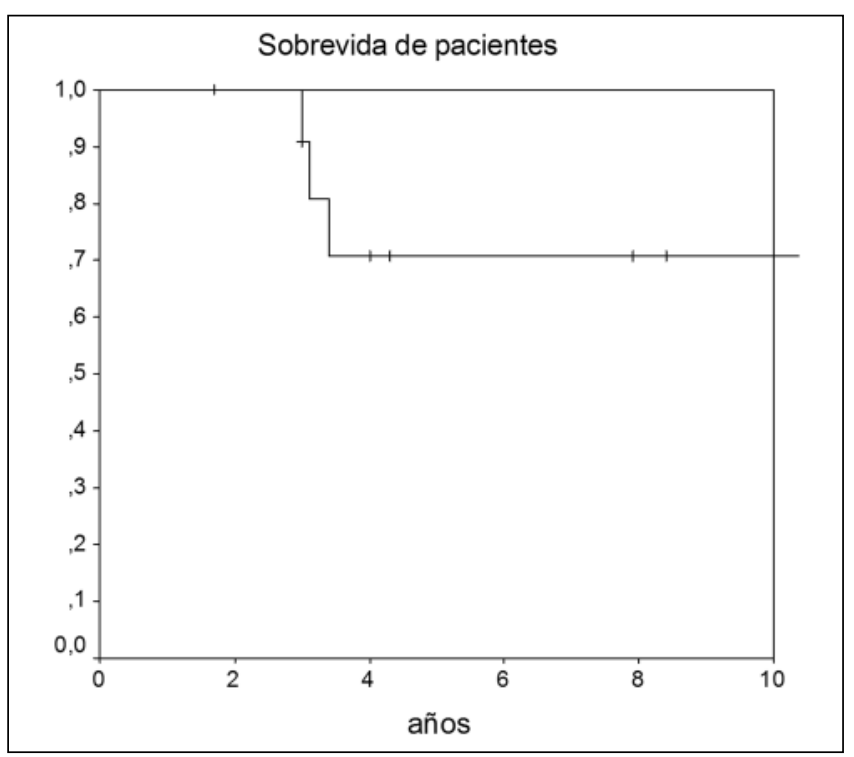

Figura 1. Sobrevida de pacientes en Trasplante Simultáneo de Páncreas y Riñón. La supervivencia acumulada es de $70 \%$ a los 5 y 10 años. Los 3 casos de fallecimiento se produjeron durante el tercer año de evolución. 
casos), 2 solamente con timoglobulina y 1 con metilprednisolona y anticuerpo monoclonal anti CD3. Dos pacientes (9 y 12 de la Tabla 3 ) presentaron rechazo agudo humoral, confirmados por la presencia de anticuerpos circulantes y C4d en la biopsia renal que fueron tratados con metilprednisolona, plasmaferesis, rituximab (Mabthera ${ }^{\circledR}$ ), inmunoglobulina en altas dosis o timoglobulina endovenosa, con normalización de la creatinina plasmática.

Tabla 3. Resultados clínicos del trasplante simultáneo de páncreas y riñón

\begin{tabular}{|c|c|c|c|c|c|c|c|c|c|}
\hline & $\begin{array}{l}\text { Año } \\
\text { de tx }\end{array}$ & $\begin{array}{l}\text { Tiempo de } \\
\text { observa- } \\
\text { ción } \\
\text { (años) }\end{array}$ & $\begin{array}{c}\text { Péptido } \\
\text { C } \\
\text { post tx }\end{array}$ & $\begin{array}{c}\text { Creat } \\
\text { post tx } \\
\text { mg/dl }\end{array}$ & $\begin{array}{l}\text { Pérdida } \\
\text { páncreas } \\
\text { (años } \\
\text { post tx) }\end{array}$ & $\begin{array}{l}\text { Pérdida } \\
\text { renal } \\
\text { (años } \\
\text { post tx) }\end{array}$ & $\begin{array}{c}\text { Rechazo } \\
\text { agudo/ } \\
\text { tratamiento }\end{array}$ & $\begin{array}{c}\text { Condición } \\
\text { del paciente } \\
\text { (diciembre } \\
\text { 2008) }\end{array}$ & $\begin{array}{l}\text { Causa de: } \\
\text { a) pérdida injerto } \\
\text { b) fallecimiento }\end{array}$ \\
\hline 1 & 1994 & 15 & 5,1 & 2,3 & - & 13 & $\begin{array}{l}\text { Celular/ } \\
\text { Timog }\end{array}$ & $\begin{array}{l}\text { Vivo } \\
\text { En diálisis }\end{array}$ & $\begin{array}{l}\text { a) Nefropatía cró- } \\
\text { nica del injerto }\end{array}$ \\
\hline 2 & 1994 & 3 & 3,3 & 1,5 & 2 & 1 & $\begin{array}{l}\text { Celular/ } \\
\text { Esteroid }\end{array}$ & $\begin{array}{l}\text { Fallecido al } \\
3 \text { año }\end{array}$ & $\begin{array}{l}\text { a) Rechazo } \\
\text { b) Cardiopatía } \\
\text { coronaria y } \\
\text { enfermedad vas- } \\
\text { cular periférica }\end{array}$ \\
\hline 3 & 1995 & 14 & 2,6 & 1,5 & - & - & $\begin{array}{l}\text { Celular/ } \\
\text { Esteroid- } \\
\text { OKT3 }\end{array}$ & $\begin{array}{l}\text { Vivo } \\
\text { sin insulina } \\
\text { sin diálisis }\end{array}$ & \\
\hline 4 & 1997 & 12 & 6,2 & 1,8 & - & - & $\begin{array}{l}\text { Celular/ } \\
\text { Esteroid }\end{array}$ & $\begin{array}{l}\text { Vivo } \\
\text { sin insulina } \\
\text { sin diálisis }\end{array}$ & \\
\hline 5 & 2000 & 9 & 5,8 & 1,1 & - & - & No & $\begin{array}{l}\text { Vivo } \\
\text { sin insulina } \\
\text { sin diálisis }\end{array}$ & \\
\hline 6 & 2001 & 3 & 9 & 1,24 & - & - & $\begin{array}{l}\text { Celular/ } \\
\text { Esteroid }\end{array}$ & $\begin{array}{l}\text { Fallecido al } \\
3 \text { año }\end{array}$ & $\begin{array}{l}\text { b) Hemiparesia } \\
\text { Pielonefritis cró- } \\
\text { nica, infección, } \\
\text { sepsis }\end{array}$ \\
\hline 7 & 2001 & 3 & 6,1 & 2,6 & 2 & 1 & $\begin{array}{l}\text { Celular/ } \\
\text { Timog }\end{array}$ & $\begin{array}{l}\text { Fallecido al } \\
3 \text { año }\end{array}$ & $\begin{array}{l}\text { a) Rechazo } \\
\text { b) Caquexia y } \\
\text { sepsis }\end{array}$ \\
\hline 8 & 2001 & 8 & 3,7 & 0,8 & - & - & No & $\begin{array}{l}\text { Vivo } \\
\text { sin insulina } \\
\text { sin diálisis }\end{array}$ & \\
\hline 9 & 2004 & 5 & & 1,1 & - & - & $\begin{array}{l}\text { Humoral/ } \\
\text { Esteroid PPF } \\
\text {-ivlg-Ritux }\end{array}$ & $\begin{array}{l}\text { Vivo } \\
\text { sin insulina } \\
\text { sin diálisis }\end{array}$ & \\
\hline 10 & 2005 & 4 & 2,8 & 0,92 & - & - & No & $\begin{array}{l}\text { Vivo } \\
\text { sin insulina } \\
\text { sin diálisis }\end{array}$ & \\
\hline 11 & 2006 & 3 & 2,5 & 1,23 & - & - & No & $\begin{array}{l}\text { Vivo } \\
\text { sin insulina } \\
\text { sin diálisis }\end{array}$ & \\
\hline 12 & 2007 & 2 & 2,3 & 1,46 & - & - & $\begin{array}{l}\text { Humoral/ } \\
\text { Esteroid- } \\
\text { Timog-Ritux }\end{array}$ & $\begin{array}{l}\text { Vivo } \\
\text { sin insulina } \\
\text { sin diálisis }\end{array}$ & \\
\hline
\end{tabular}

tx: trasplante. 

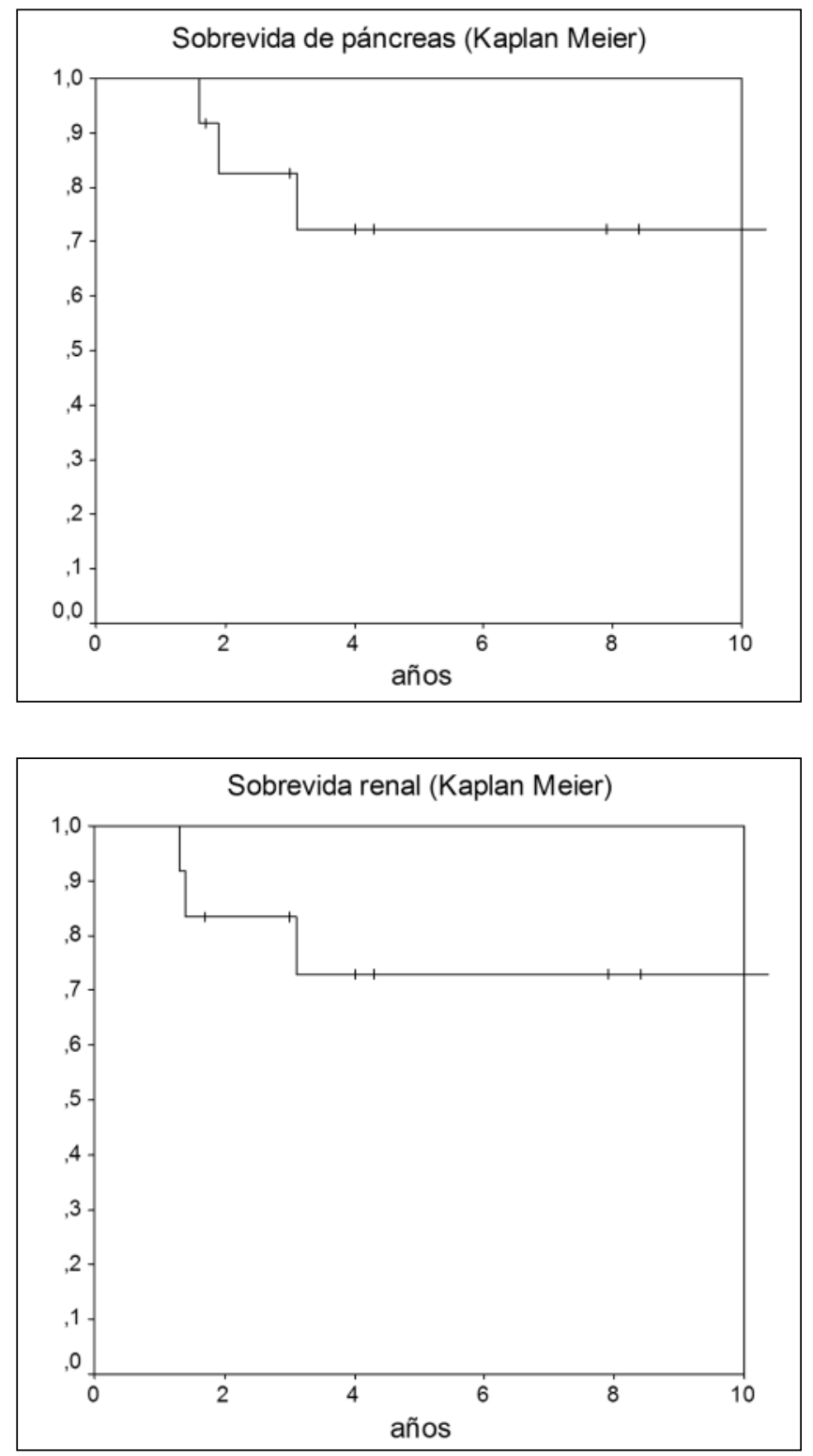

Figura 2. Sobrevida actuarial de páncreas definido como independencia de insulina. El fallecimiento con páncreas funcionante fue considerado como falla del páncreas.

Figura 3. Sobrevida de injertos renales. El fallecimiento con riñón funcionante fue considerado como falla renal. Tres de los 12 pacientes tienen un tiempo de observación superior a los diez años.

\section{Complicaciones vasculares}

Cuatro pacientes tuvieron complicaciones vasculares. El paciente 2 tuvo una enfermedad vascular difusa de vaso grueso y pequeño, pérdida funcional de ambos injertos, angioplastía coronaria y amputación en el pié derecho. La paciente 5 requirió amputación a nivel del metatarso. El paciente 6 tuvo un infarto medular lumbar, secuela neurológica permanente e isquemia y amputación del pie derecho. La paciente 7 requirió de amputación infracondílea.

\section{Complicaciones infecciosas}

En 6 de los 12 pacientes se trataron y curaron las siguientes infecciones severas: artritis estafilocócica secundaria a sepsis, criptocococis intestinal, osteomielitis del calcáneo secundaria a micosis sistémica, mucormicosis sinusal, dos casos de enfermedad por CMV. Dos pacientes murieron por cuadros sépticos: un caso de sepsis relacionada a una escara sacra recurrente y pielonefritis aguda recidivante por vejiga neurogénica (paciente 6) y otro por pielonefritis micótica (paciente 7). 
Trasplante de páncreas y riñón en diabéticos - A. Alba et al

\section{Otras complicaciones}

Un paciente presentó colecistitis aguda tratada con colecistectomía. Un receptor presentó pancreatitis aguda del injerto que respondió a tratamiento médico. En 2 receptores se presentó un síndrome febril atribuido al anticuerpo monoclonal alemtuzumab.

No se presentaron enfermedades neoplásicas.

En los 9 pacientes con función pancreática endocrina adecuada, no se observó progreso en la oftalmopatía diabética ni neuropatía periférica, $y$ realizaron sus actividades laborales en forma normal.

\section{Discusión}

Los pacientes portadores de nefropatía diabética presentan elevadas tasas de mortalidad tanto en el período de insuficiencia renal previo a la diálisis como durante este tratamiento ${ }^{5}$. Los pacientes diabéticos tipo 1 se benefician del TSPR, lo que mejora la sobrevida y la calidad de vida. El TSPR ha experimentado cambios que han contribuido a optimizar sus resultados: abocamiento del páncreas al intestino delgado ${ }^{6,7}$; menores efectos adversos de la inmunosupresión por reducción y eliminación de la terapia esteroidal ${ }^{8}$, incorporación de micofenolato mofetil y tacrolimus ${ }^{9}$, inducción con timoglobulina o alemtuzumab ${ }^{10}$; determinación periódica de anticuerpos anti HLA y vigilancia frecuente en el riñón trasplantado con biopsia. El programa de TSPR de Clínica Las Condes ha aplicado estas tecnologías y esta comunicación presenta los resultados obtenidos 15 años después del primer TSPR ${ }^{11}$.

En concordancia con resultados obtenidos en grandes centros ${ }^{12-14}$, en esta experiencia la sobrevida de pacientes fue de $70 \%$, la sobrevida de páncreas $72 \%$ y la sobrevida de riñón fue de $73 \%$ en 10 años cifras superiores a la sobrevida de diabéticos en diálisis en lista de espera de trasplante, que es $46 \%$ a cuatro años ${ }^{15}$. Al final del período de observación 9 pacientes estaban euglicémicos en ayunas, presentaban niveles normales de Péptido $\mathrm{C}$ e independencia de insulina o hipoglicemiantes orales, independencia de diálisis en 8 pacientes. En este estudio no se efectuó un análisis prospectivo de la calidad de vida de los receptores, sin embargo, los 9 pacientes con páncreas funcionantes realizan actividades normales, no presentaron eventos cardiovasculares graves ni deterioro mayor de la retinopatía, lo que coincide con la información disponible en diversas publicaciones s, $^{46}$.

En la literatura, los criterios de selección de receptores incluyen descartar a pacientes mayores de 50 años, con patología cardiovascular irreparable, neuropatía y/o oftalmopatía avanzada, además de las contraindicaciones generales de trasplante (cáncer, enfermedades infecciosas activas etc). Estos criterios deben ajustarse a un análisis caso a caso como lo demuestra la estadística de la United Network for Organ Sharing (UNOS, USA) con $15 \%$ de mayores de 50 años en receptores de TSPR ${ }^{17}$. En la presente experiencia la edad promedio de los receptores al momento del trasplante fue de 38,9 años; la distribución igual por género, tiempo promedio de evolución de la DM1 fue de 22 años, el tiempo promedio de espera en diálisis fue de 10 meses. En esta cohorte la mayoría de los pacientes se trasplantó con enfermedad vascular periférica y cardiopatía coronaria severas, lesiones que explican la morbilidad y mortalidad en el post trasplante. Por lo tanto, los resultados podrían optimizarse en pacientes que se trasplantaran antes de tener secuelas avanzadas. Las complicaciones inmunológicas, que tuvieron una alta incidencia (8 pacientes), no incidieron en la pérdida de injertos, pero impactaron la filtración glomerular.

La experiencia extranjera señala que el TSPR tiene la mejor relación costo beneficio y constituye la mejor alternativa de tratamiento para pacientes con DM 1 e insuficiencia renal terminal ${ }^{18}$.

En nuestro medio no se han efectuado dos técnicas alternativas al TSPR: trasplante de páncreas en receptor ya trasplantado de riñón y trasplante aislado de páncreas en sujetos con función renal mayor de $50 \mathrm{ml} / \mathrm{min}^{19,20}$. La primera opción requiere un programa de procuramiento de páncreas desarrollado y expedito, mientras la segunda opción se aplica selectivamente a DM 1 muy inestable y las consideraciones de los riesgos de la inmunosupresión han prevalecido por sobre las ventajas de la euglicemia.

En conclusión, la experiencia de un centro en TSPR aplicado a receptores con DM1 e insuficiencia renal crónica terminal demuestra resultados que aconsejan incrementar el TSPR para este tipo pacientes, prefiriendo esta terapia a la sustitución funcional con diálisis o con trasplante renal aislado. 


\section{Referencias}

1. García de los Ríos M. Diabetes Mellitus. Libro editado por la Fundación de Investigación y Perfeccionamiento Médico. Santiago de Chile, 2003.

2. Pambianco G, Costacou T, Ellis D, Becker DJ, Klein $\mathrm{R}$, Orchard TJ. The 30-year natural history of type 1 diabetes complications: the Pittsburgh Epidemiology of Diabetes Complications Study experience. Diabetes 2006; 55 (5): 1463-9.

3. Sanhueza ME, Cotera A, Elgueta L, López SG, Loncon P, Macan F, et al. Diabetes y hemodiálisis. Caracterización de una cohorte y seguimiento a cuatro años. Rev Med Chile 2008; 136: 279-86.

4. Andreoni KA, Brayman KL, Guidinger MK, Sommers CM, Sung RS. Kidney and Pancreas Transplantation in the United States, 1996-2005. Am J Transplant 2007; 7 (Part 2): 1359-75.

5. Fierro JA, Nefropatía diabética I: Fisiopatología, diagnóstico, evolución clínica y epidemiologia. Rev Med Clin Condes 2009; 20 (5): 639-50.

6. Stephanian E, Gruessner RW, Brayman KL, Gores P, Dunn DL, Najarian JS, et al. Conversion of Exocrine Secretions From Bladder to Enteric Drainage in Recipients of Whole Pancreaticoduodenal Transplants. Ann Surg 1992; 216: 663-72.

7. Monroy-Cuadros M, Salazar A, Yilmaz S, McLaughlin $\mathrm{K}$, et al. Bladder vs enteric drainage in simultaneous pancreas-kidney transplantation. Nephrol Dial Transplant 2006; 21: 483-7.

8. Rajab A, Pelletier RP, Ferguson RM, Elkhammas EA, Bumgardner GL, Henry ML. Steroid-free maintenance immunosuppression with rapamune and low-dose neoral in pancreas transplant recipients. Transplantation 2007; 84 (9): 1131-7.

9. Saudek F, Malaise J, Boucek P, Adamec M; Euro-SPK Study Group. Efficacy and safety of tacrolimus compared with cyclosporin microemulsion in primary SPK transplantation: 3-year results of the Euro-SPK 001 trial. Nephrol Dial Transplant 2005; 20 (Suppl 2): ii3-10, ii62.

10. Farney A, Sundberg A, Moore P, Hartmann E, Rogers J, Doares W, et al. A randomized trial of alemtuzumab vs anti-thymocyte globulin induction in renal and pancreas transplantation. Clin Transplant 2008; 22 (1): 41-9.

11. Buckel E, Morales J, Aguiló J, Rosenfeld R, Pefaur J, Zavala C, et al. [Simultaneous pancreas-kidney transplantation. First cases in Chile]. Rev Med Chile 1996; 124 (1): 83-8.

12. Ojo AO, Meier-Kriesche HU, Hanson JA, Leichtman A, Magee JC, Cibrik D, et al. The impact of simultaneous pancreas-kidney transplantation on long-term patient survival. Transplantation 2001; 71: 82-90.

13. Becker BN, Brazy PC, Becker YT, Odorico JS, Pintar TJ, Collins BH, et al. Simultaneous pancreas-kidney transplantation reduces excess mortality in type 1 diabetic patients with end-stage renal disease. Kidney Int 2000; 57 (5): 2129-35.

14. Tydén G, Bolinder J, Solders G, Brattström C, Tibell A, Groth CG. Improved survival in patients with insulindependent diabetes mellitus and end-stage diabetic nephropathy 10 years after combined pancreas and kidney transplantation. Transplantation 1999; 67 (5): 645-8.

15. White SA, Shaw JA, Sutherland DE. Pancreas transplantation. Lancet 2009; 23; 373 (9677): 1808-17.

16. de Sá JR, Monteagudo PT, Rangel EB, Melaragno CS, Gonzalez AM, Linhares MM, et al. The evolution of diabetic chronic complications after pancreas transplantation. Diabetol Metab Syndr 2009; 1: 11.

17. La Rocca E, Fiorina P, Astorri E, Rossetti C, Lucignani G, Fazio F, et al. Patient survival and cardiovascular events after kidney-pancreas transplantation: comparison with kidney transplantation alone in uremic IDDM patients. Cell Transplant 2000; 9 (6): 929-32.

18. Douzdjian V, Ferrara D, Silvestri G. Treatment strategies for insulin-depndent diabetics with ESRD: a cost-effectiveness decision analysis model. Am J Kidney Dis 1998; 31 (5): 794-802.

19. Farney AC, Borboroglu PG, Young C, et al. Native renal function after pancreas transplant alone. JASN 2002; 13: 188.

20. Coppelli A, Giannarelli R, Vistoli F, Del Prato S, Rizzo G, Mosca F, et al. The beneficial effects of pancreas transplant alone on diabetic nephropathy. Diabetes Care 2005; 28: 1366-70. 